\title{
STUDY OF 4D PRIMITIVES' SELF-TRANSFORMATION
}

\author{
Matej Pivar (D), Deja Muck (D) \\ University of Ljubljana, Faculty of Natural Sciences and Engineering, Ljubljana, Slovenia
}

\begin{abstract}
D$ printing is the process through which a $3 D$ printed object or primitive is transformed into another structure under the influence of external energy input such as temperature, light or other extertal stimuli. The 4th dimension is the time in which the primitive changes its appearance. In most cases, the shape changes. We call this a self-assembly or self-transformation process.

In the process of printing a primitive, capable of transforming themselves from one shape to another, we often encounter combinations of one or two thermoplastic materials that have different thermal and physico-mechanical properties. The printed primitive is transformed where the active element is contained. The active element is the basic building block of the self-transforming primitive. For this purpose, it is necessary to choose the appropriate combination of thermoplastic materials, to determine the length of the active element and the number of layers of which it is composed. For the printing of the active element two thermoplastic materials can be selected which differ from each other in their thermal transitions and physico-mechanical properties. The process of transformation under the influence of elevated temperature should be carried out in such a way that the printed primitive is heated above the temperature of the glass transition that the material used on the active elements has. This releases the residual stresses created during the printing process and causes the active material to shrink. In this way, a primitive can be transformed from a flat shape to a final 3D shape. This shape is then maintained by controlled cooling below the glass transition temperature of the active element.

In this paper the first research results of the primitive transformation were presented. The appropriate combination of materials and the optimal temperature of the water as external stimuli were determined, and finally the primitives' shape recovery. In the research we used the active element which consists of a single layer of flexible, elastic thermoplastic material (passive material) and three layers of thermoplastic materials with the properties of shape memory polymers (active material). For printing we used the multitool 3D printer ZMorph which is based on Fused Deposition Modeling (FDM) technology.
\end{abstract}

Key words: 3D printing, shape memory polymers, 4D printing, primitive, self-transformation

\section{INTRODUCTION}

3D printing is already a well-known and widespread field of application. It is an additive technology, producing a three-dimensional object from a digital model using various technologies. Recently, research in the field of 3D printing has increasingly focused on the use of functional materials. These materials allow 3D printed objects to change over time as a fourth dimension. This creates a new area of use for 3D printing, called 4D printing. Printed objects are capable of independently transforming or changing chemical or physical properties under the influence of various external stimuli. The changes can be reversible or irreversible and thus open new areas of application in biomedicine, robotics, architecture, construction, textiles, etc. (Shie et al, 2019).

One of the simplest options for $4 \mathrm{D}$ printing applications is the use of thermoplastic materials that are capable of thermoforming or thermal transformations. This group of thermoplastic materials also includes stimuli-responsive polymers, also known as shape memory polymers. They can change their shape and have the ability to regain their original shape (Shie et al, 2019).

A big advantage of thermoplastic materials is that they can be printed with fused deposition modeling. This technology is simple and affordable. In the process of printing a primitive that will be able to transform, we mostly encounter one or a combination of two thermoplastic materials. In the first case, using one material, the transformation is controlled by a different orientation of the deposited filament layers of the active material (Teunis et al, 2017). In the second case, the transformation is achieved by the right combination of thermoplastic materials, which differ in thermal transitions and have different physical and mechanical properties. For active parts of the primitive, a combination of active and passive material is used. Active material shrinks when heated above its glass transition temperature $(\mathrm{Tg})$ and below its melting temperature (Tm). Passive material does not change its shape at the same temperature but serves as support for the active material to transform into a specific direction. For the passive part of the primitive, we only use the passive material (Kačergis et al, 2019). 
In order to control the transformation, it is important to properly prepare the material for printing. Polymers are hygroscopic (Kačergis et al, 2019; Tham et al, 2016; Zaldivar et al, 2018; Valeraga et al, 2018), so the filaments must be dried before printing. In this way we achieve good printing quality. Moisture can affect the extrusion process, filament deposition and the mechanical properties of printed primitives (Almenara, 2017). Due to the presence of moisture during the printing process, air pockets can form within the printed primitive (Penjumras et al, 2015; Byoungkwon et al, 2018; Guanyun et al, 2018). This has a negative impact on transformation.

The transformation depends on the residual stress created during the 3D printing process. During the extrusion process the thermoplastic materials transition to a highly elastic state. The polymer chains are forcibly oriented in the longitudinal direction of the material flow through the extrusion nozzle. As soon as material leaves the nozzle, it begins to cool and solidify, forcing the chains to maintain their rearranged state.

When the 3D object is reheated above the glass transition temperature of the active thermoplastic material, the polymer chains begin to reorient themselves back into their chaotic, or low energy mode, causing shrinkage along the printed direction and changing the shape of the 3D printed object (Teunis et al, 2017; Kačergis et al, 2019; João et al, 2018; Uzir et al, 2015).

The printing speed and the speed of cooling play a very important role in the transformation of primitives. In literature (Kačergis et al, 2019; João et al, 2018; Song and Cassandra, 2016), it has been shown that higher transformation is achieved higher with printing speed. On the other hand, the higher the printing speed, the lower the printing accuracy and the worse the mechanical properties along with visual quality (Kadir and Türkmen, 2018; Singh, 2019; Ravichandra and Shanmugam, 2018). In case of excessive cooling, the layers of filaments will not merge with each other and stratification will occur (Kadir and Türkmen, 2018). However, if the cooling is insufficient, the quality of 3D printing is poorer, as the pre-deposited layer is not strong enough for the deposition of new layers.

To control the described transformation, it is first necessary to optimize the printing conditions. Our research presents the most important parameters that we must optimize before printing. Besides the printing conditions, we have also determined the appropriate combination of thermoplastic materials and the optimal temperature of the activation medium. In this way we create good conditions for the study of the self-transformation of primitives, based on which we intend to establish a mathematical model to predict the level of the transformation and the primitives' shape recovery.

\section{METHODS}

\subsection{Materials}

Thermoplastic materials in the form of filaments (diameter $1.75 \mathrm{~mm}$, manufactured by Plastika Trček d.o.o. (Slovenia)) were used, differing in their thermal and physico-mechanical properties. For the printing of the passive part of the primitive we used TPU thermoplastic materials with two different hardnesses, named 85 and 89 according to the Shore A scale (hereinafter referred to as TPU80 and TPU90). For printing the active part of the primitive, we used a combination of thermoplastic materials, TPU80 and PLA in the first case and TPU90 and PLA in the second. To achieve a precise and reproducible transformation the materials were dried before printing.

\subsection{Modeling of the primitives}

The modeling of primitives for the study of self-transformation was carried out in the Blender software (Blender Institute, Netherlands). To prepare the models for 3D printing we used the software Slic3r (RepRap Community).

\subsection{Optimization of the printing conditions}

In the research we used a multitool 3D printer ZMorph VX (ZMorph S.A., Poland), which allows printing with two thermoplastic materials. To achieve a reproducible and accurate primitive transformation we first have to calibrate the printer and then optimize the printing conditions. The most important thing is to optimize the printing speed, flow rate and cooling rate of the extruded material. 


\subsubsection{Printer calibration}

The calibration of the 3D printer includes the leveling of the work platform and the adjustment of the offset of the extrusion nozzles from the work platform. The build platform was manual leveled using a ZMorph probe.

The nozzle offset was adjusted by printing a $200 \mu \mathrm{m}$ thick layer. The corresponding nozzle offset was confirmed when the thickness of the printed layer reached $200 \mu \mathrm{m}$.

\subsubsection{Printing speed}

The printing speed affects the size of the final transformation. In order to eliminate as many factors that influence the transformation as possible, we have unified the speeds of both (active and passive) materials to the maximum printing speeds of TPU materials. Therefore, when producing test samples or primitives, we have used two different printing speeds, $15 \mathrm{~mm} / \mathrm{s}$, which corresponds to the maximum printing speed of the softer thermoplastic TPU80, and the speed of $22 \mathrm{~mm} / \mathrm{s}$, which corresponds to the maximum printing speed of the thermoplastic TPU90.

\subsubsection{Flow rate}

By varying the ratio of printing speed to extrusion speed, a smaller or larger amount of material can be extruded. To achieve precise and reproducible transformation, the deposition of filaments in the layers must be as uniform as possible and without overlapping or deformation.

This is achieved by determining the extrusion width and the flow rate. The extrusion width, used in the study, was left as a default value. The flow rate was controlled by an extrusion multiplier which adjusts the extrusion and the printing speed.

The flow rate was determined separately for both materials at a given extrusion temperature and printing speed. It was determined based on the printing of a 3D test object $(5(X) \times 50(Y) \times 2(Z) \mathrm{mm})$. We used a linear infill pattern of $100 \%$ density, oriented with the longer side in a longitudinal direction. Finally, we also created a 3D test object of the layered sandwich structure of both materials and analyzed the stacks when one material was deposited onto the other. Two extrusion nozzles of the print head were active simultaneously. After printing, the test 3D object was cross-cut and images were taken with a Dino-lite AM4113ZT optical microscope. We captured the photos at 50x magnification.

\subsubsection{Cooling the extruded material}

As soon as the polymer leaves the extrusion nozzle it begins to cool and solidify. Cooling is very important to achieve good visual quality of 3D printed objects and their good mechanical properties. Too much cooling leads to delamination, while insufficient cooling leads to poor visual quality and loss of detail, since the previously deposited layer is not strong enough to carry a new one.

The cooling speed with the fans has always been set by the manufacturer to a constant average recommended speed. In order to control the cooling as much as possible and to eliminate the influence of the room air temperature, we have placed the printer in a closed housing.

\subsection{The study of self-transformation}

After optimization of printing conditions, the research was focused on the effect of material combinations and the temperature of the water as external stimuli on the self-transformation on primitives. Finally, the primitives' shape recovery was studied. Primitives were printed in dimensions $10(\mathrm{X}) \times 60(\mathrm{Y}) \times 0,8(\mathrm{Z}) \mathrm{mm}$, with two lengths of active elements, 10 and $15 \mathrm{~mm}$ (Figure 1).

The active part of the primitive consisted of three layers of active PLA material and one layer of passive TPU material. The passive part of the test 3D model consisted of four layers of TPU material (Figure 1). The infill pattern was linear in longitudinal direction. As stated before, two different TPU materials, with different hardnesses were used. In the first case, the TPU80, and in the second, the TPU90. Testing of the transformation in hot water was performed in a bath with controlled heating, which was maintaining the water temperature at $\pm 1^{\circ} \mathrm{C}$. The transformation was tested at temperatures: $60,70,80$ and $90^{\circ} \mathrm{C}$. Three test specimens were tested at each temperature. The magnitude of the transformation was determined indirectly by measuring the angle of transformation (Figure 2) as a function of time. The opposite angle was calculated from the measured angle. 


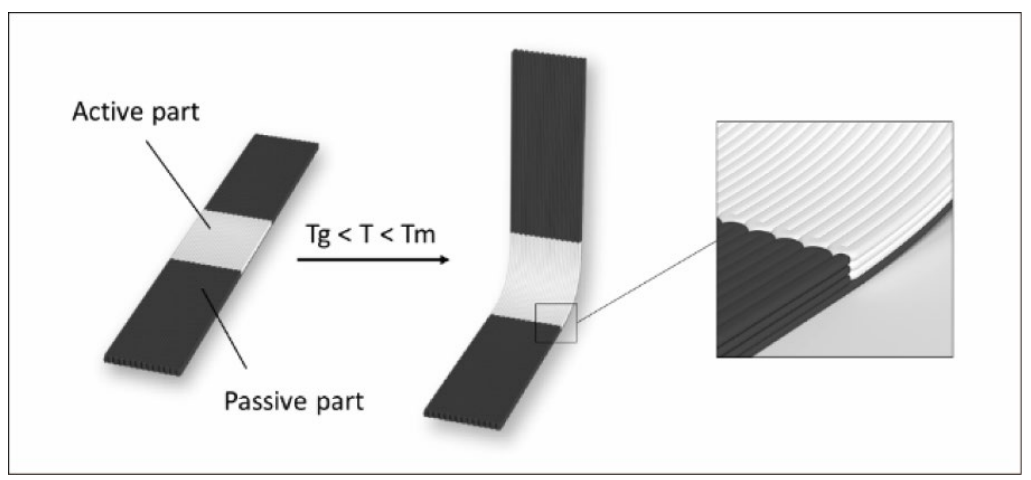

Figure 1: Schematic presentation of printed primitive and its self-transformation

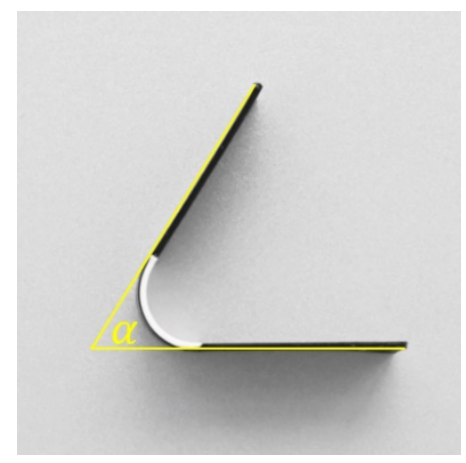

Figure 2: Determining the angle of primitives' transformation

\subsubsection{Determining the appropriate combination of materials}

In this part of the research, the combination of thermoplastic materials for the production of the active part of the primitive, was determined. In the first case, a combination of PLA and a softer TPU80 thermoplastic was used for the active part and in the second case PLA with a harder TPU90.

The active parts of the primitive had two different lengths $10 \mathrm{~mm}$ and $15 \mathrm{~mm}$. The transformation was analyzed in hot water at temperatures of 70 and $80^{\circ} \mathrm{C}$.

\subsubsection{Determination of the optimal water temperature}

The temperature of the water affects the rate and size of the primitive transformation. The higher the temperature, the faster the transformation and vice versa, the lower the temperature, the slower the transformation. The aim of our research was to determine the optimal temperature to which we need to heat water in order to perform an optimal and controlled transformation of the primitives. Primitives with an active part of $15 \mathrm{~mm}$ in length were used in the research. The transformation was tested at a temperature of $60,70,80$ and $90^{\circ} \mathrm{C}$.

\subsubsection{Determination of primitives' shape recovery}

At the end of the research, the primitives' shape recovery was determined. The primitives were produced with a combination of TPU90/PLA materials. The experiments were performed in hot water at $70^{\circ} \mathrm{C}$ (Figure 3). Primitives with an active part of $15 \mathrm{~mm}$ in length were used. The test were performed in five cycles of heating, transformation into the original form by means of force and temperature and reheating. 


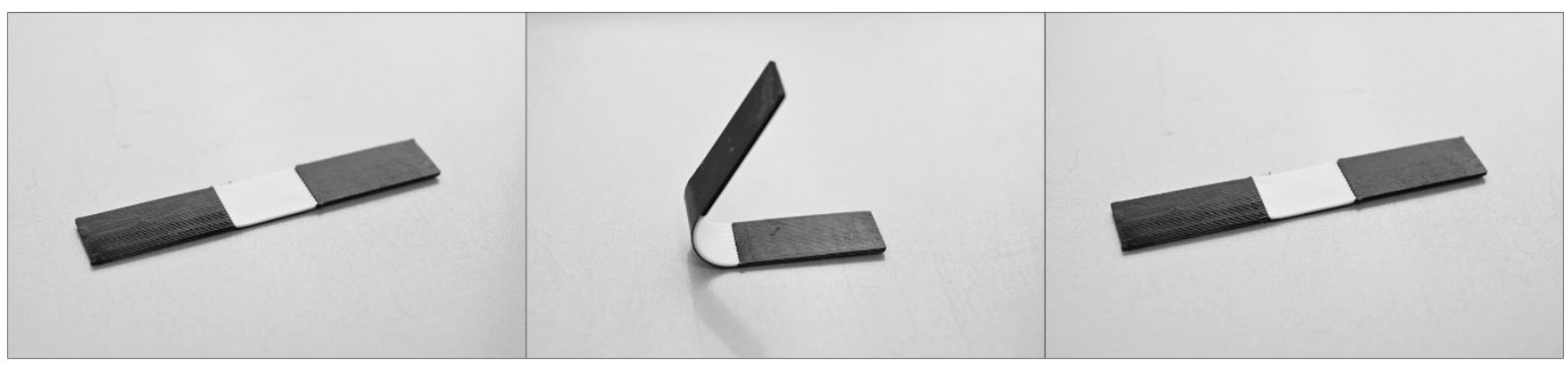

Figure 3: Shape recovery cycle of printed primitive; Phase 1 (left) - printed flat structure, Phase 2 (center) - selftransformation in hot water, Phase 3 (right) - transformation with force and hot water back into flat structure

\section{RESULTS}

\subsection{Optimization of printing conditions}

When optimizing the printing conditions, the appropriate printing speed, flow and cooling rate of the extruded material were determined. Optimal settings were set through the printing of primitives and their cross-sectional analysis (Table 1).

Table 1: Examples of unsuitable and suitable depositions of individual materials and simultaneous deposition of two thermoplastic materials

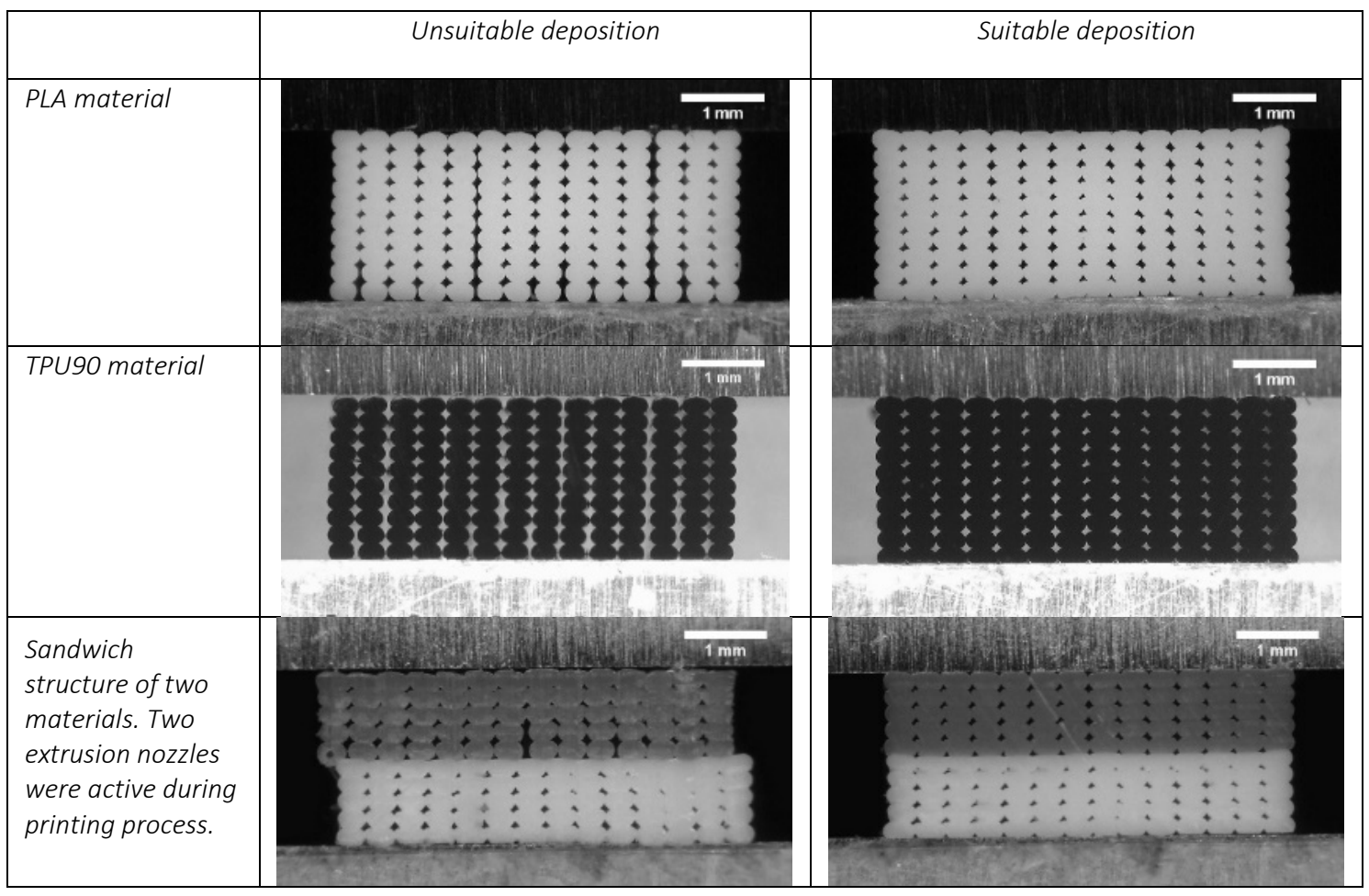

\section{2. Determining the appropriate combination of materials}

Primitives with an active part of 10 and $15 \mathrm{~mm}$ in length were printed at a printing speed of $15 \mathrm{~mm} / \mathrm{s}$. This is the maximum printing speed of the softer TPU80 thermoplastic material. A combination of TPU80/PLA and TPU90/PLA materials was used in printing of the active part. The self-transformation was tested in hot water first at a temperature of $70^{\circ} \mathrm{C}$ and $80^{\circ} \mathrm{C}$. At each temperature three primitives were tested. The results are given in Figures 4 and 5. 


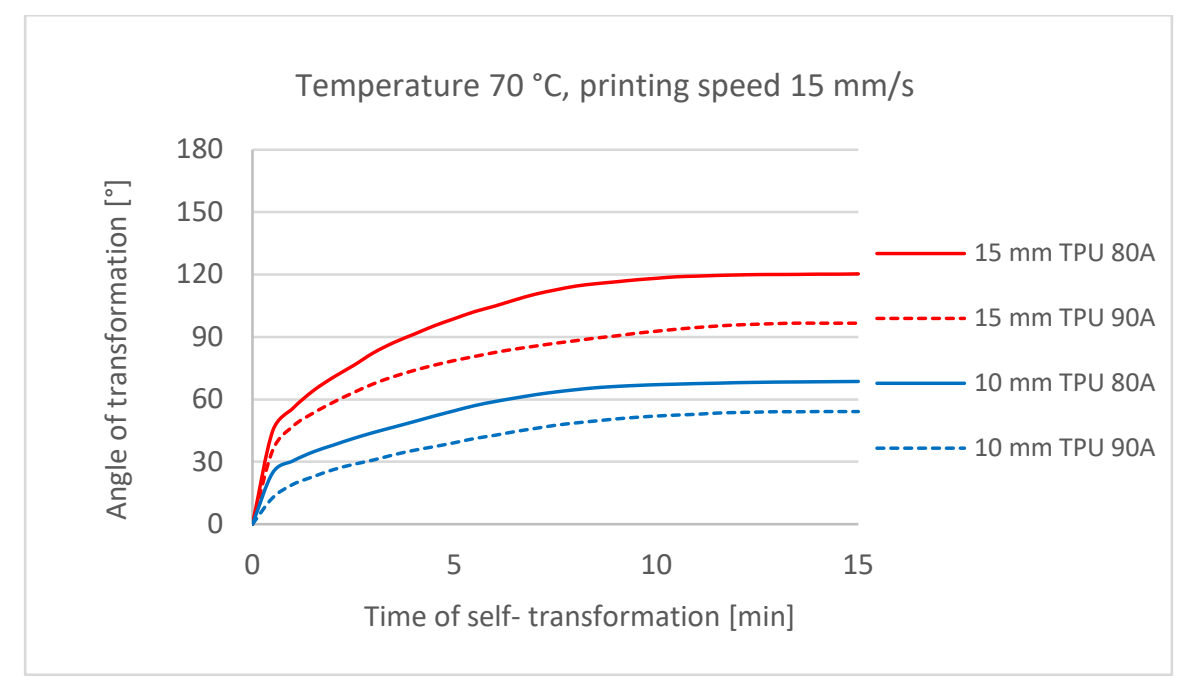

Figure 4: Angle of transformation of the primitives' (PLA/TPU80 and PLA/TPU90) depending on time in hot water at $70{ }^{\circ} \mathrm{C}$. The lengths of active part were 10 and $15 \mathrm{~mm}$. Primitives were printed at $15 \mathrm{~mm} / \mathrm{s}$

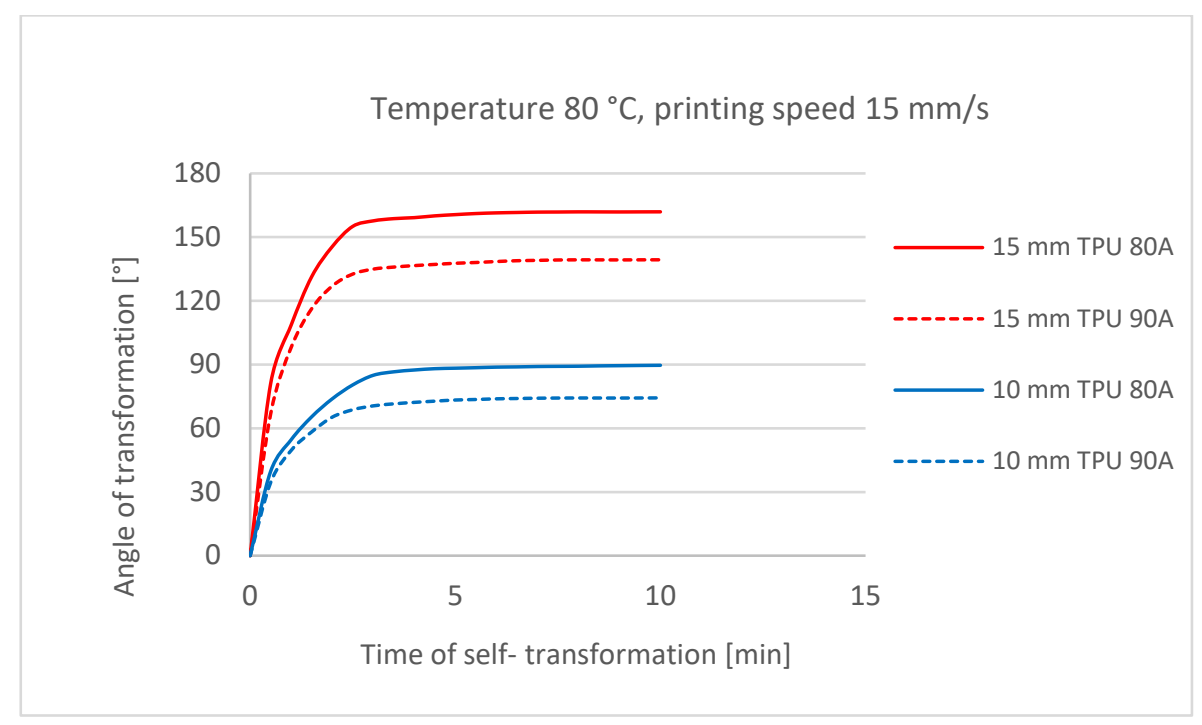

Figure 5: Angle of transformation of the primitives' (PLA/TPU80 and PLA/TPU90) depending on time in hot water at $80{ }^{\circ} \mathrm{C}$. The lengths of active part were 10 and $15 \mathrm{~mm}$. Primitives were printed at $15 \mathrm{~mm} / \mathrm{s}$

\subsection{Determining the optimal water temperature}

In this part of the research the primitives with combination of PLA and TPU90 thermoplastic materials were printed at printing speed of $22 \mathrm{~mm} / \mathrm{s}$. The printing speed was increased from 15 to $22 \mathrm{~mm} / \mathrm{s}$ to achieve higher transformations. The length of active part was $15 \mathrm{~mm}$. To determine the optimal temperature water heated to $60,70,80$ and $90{ }^{\circ} \mathrm{C}$ was used. Results are presented in Figure 6. 


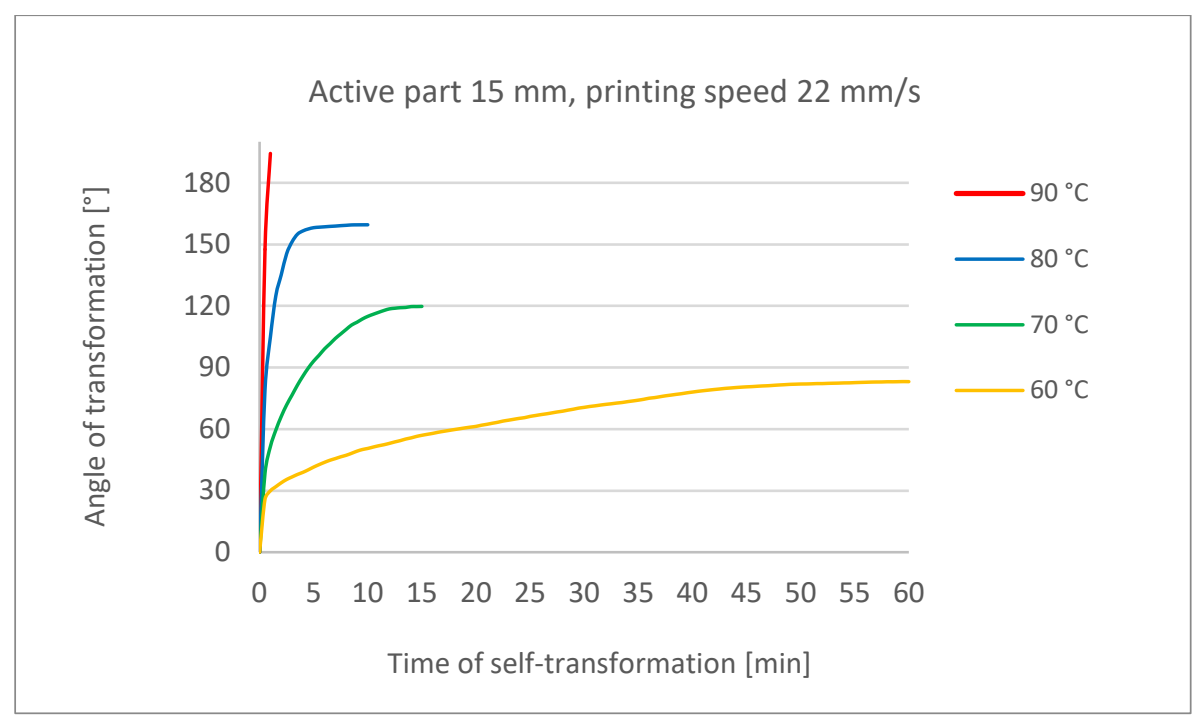

Figure 6: Angle of transformation of the primitives' (PLA/TPU90) depending on time in heated water at 60, 70, 80 and $90{ }^{\circ} \mathrm{C}$. The length of active part was $15 \mathrm{~mm}$. Primitives were printed at $22 \mathrm{~mm} / \mathrm{s}$

\subsection{Determination of primitives' shape recovery}

Testing of primitives' shape recovery response was performed on primitives immersed in hot water at $70^{\circ} \mathrm{C}$. The length of primitives' active part was $15 \mathrm{~mm}$. Firstly, the structures were subjected to four additional self-transformation cycles, i.e. they were heated and placed in a flat position, cooled and then immersed in hot water again to allow self- transformation. This process was repeated four times to increase the total number of transformation cycles to five. The results are visible in Figure 7.

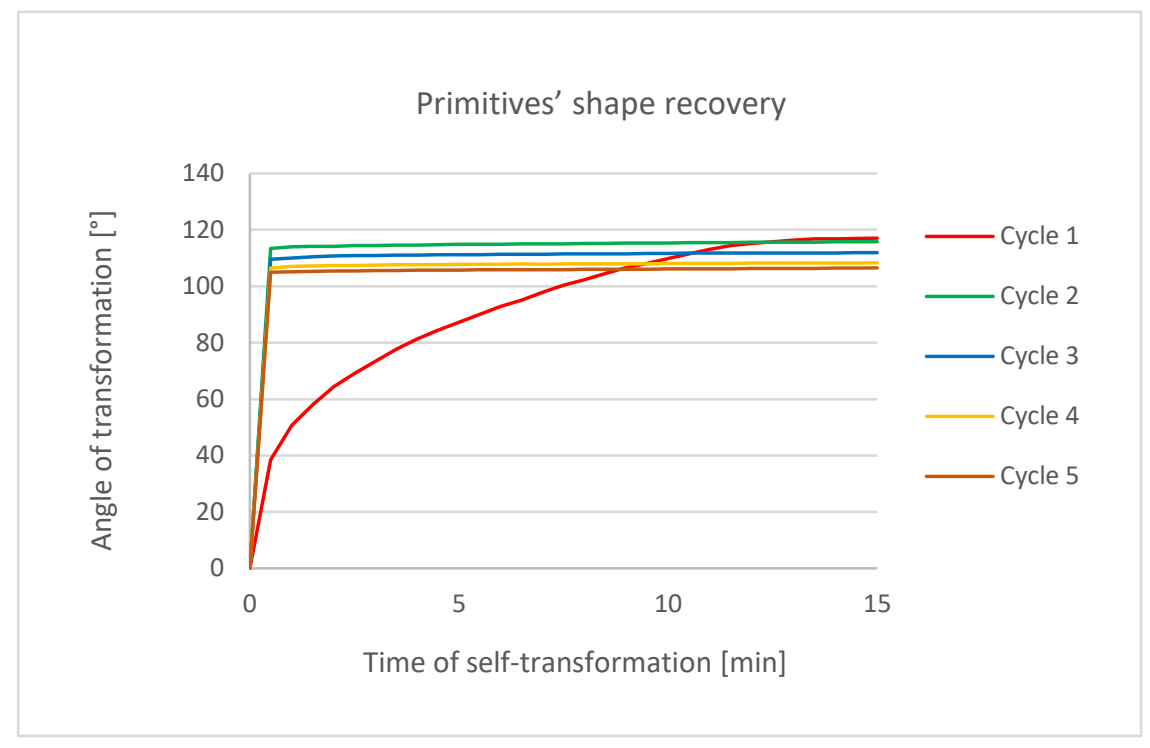

Figure 7: Results of primitives' (TPU90/PLA) shape recovery. Primitives were subjected to five self-transformation cycles in hot water at $70^{\circ} \mathrm{C}$

\section{DISCUSSION}

For a successful study of primitives' self-transformation processes, a calibrated printer and optimized printing conditions are needed. If we want to achieve a precise and controllable transformation, the deposition of filaments in layers must be even, without overlap and deformation. Layers of passive and active material printed on top of each other must achieve adequate deposition and good adhesion. This is presented in the images in Table 1. 
When determining the appropriate combination of materials, we came to the following conclusions (Figures 4 and 5). The rate of transformation decreases over time. The maximum transformation angle increases with the length of the active part of the primitive and with increasing temperature of water. By raising the activation temperature, the maximum angle of transformation is reached in a shorter time. At the temperature of $70^{\circ} \mathrm{C}$, the transformation stops within 15 minutes, and at the temperature of $80^{\circ} \mathrm{C}$ in less than 10 minutes.

If we compare primitives made of different combinations of materials, we can state the following. The maximum angle of transformation for a combination of PLA materials and softer TPU80 is higher than the transformation angle for a combination of PLA materials and harder TPU90 thermoplastic at every temperature. We had problems with extrusion of the TPU80 material. As the temperature rises, it's viscosity becomes very low, completely liquid, which causes contamination of the PLA layers of the active part. Thus, it negatively affects the transformation of the primitive. Due to these problems, we had to exclude TPU80 from the study and proceed only with the harder TPU90 thermoplastic.

In determining the optimal temperature of the activation medium, we found out that a higher activation temperature increases the rate and angle of the transformation of primitives (Figure 6). At an activation temperature of $60^{\circ} \mathrm{C}$, the transformation does not stop after 60 minutes, and at $90^{\circ} \mathrm{C}$ it is completed within 2 minutes. Due to the slow and low response of the primitives at an activation temperature of $60^{\circ} \mathrm{C}$, this temperature was excluded from further studies. We also excluded the $90^{\circ} \mathrm{C}$ temperature do to it being very difficult to control. Temperatures of 70 and $80{ }^{\circ} \mathrm{C}$ proved to be the most suitable for primitive transformation. In the end, we decided to conduct further research at a lower temperature, i.e. $70^{\circ} \mathrm{C}$. The decision is argued by the fact that the energy consumption for heating the water is lower, and the time response of primitives can be well analyzed.

From the results of the shape recovery given in Figure 7, the following conclusions can be deduced. In the first cycle, at a water temperature of $70{ }^{\circ} \mathrm{C}$, the transformation takes 15 minutes. The polymer chains orient themselves in their favorable position and when they are fully released (shrunk) the transformation stops. With each subsequent cycle, the transformation is almost completely performed in the first 2 seconds. The transformation is faster, since the chains are already oriented to their favorable position. They shrink back only in the direction in which we stretched them. With each subsequent cycle, the final transformation angle decreases by an average of $2-3^{\circ}$.

\section{CONCLUSIONS}

The following conclusions can be drawn from the conducted research. For a successful study of 4D primitives' self-transformation we must ensure optimal printing conditions, select the appropriate combination of materials and the optimal temperature of the water as external stimuli. We found that in addition to printer calibration it is necessary to determine the appropriate printing speed, flow and cooling rate of the material. Since the active part of the primitive consists of two different materials it is important that the sandwich structure of materials is properly deposited and has achieved a good adhesion between the layers. The size of the transformation is also influenced by the passive material used on the active part of the primitive since it provides support during the transformation. If its hardness is insufficient and is too flexible, the transformation may become less precise. We also found out that the size of the transformation is influenced by the length of the active part of the primitive, the printing speed and the temperature of the hot water. A higher angle of transformation is achieved when the active part is longer and when the printing speed and the temperature of the water are higher.

In our case, we can conclude that for quality and controlled transformation it is recommended to make primitives with a length of the active part of $15 \mathrm{~mm}$, to use max. print speed $22 \mathrm{~mm} / \mathrm{s}$ and hot water as external stimuli at $70{ }^{\circ} \mathrm{C}$. This is the basis on which we can make a mathematical model for predicting the primitives' self-transformation and a model for predicting the primitives' shape recovery.

\section{REFERENCES}

[1] Almenara, C. C. I.: "Anisotropy and Humidity Effect on Tensile Properties and Electrical Volume Resistivity of Fused Deposition Modeled Acrylonitrile Butadiene Styrene Composites", PhD thesis, Pontificia Universidad Catolica del Peru-CENTRUM Catolica (Peru), 2017. 
[2] Byoungkwon, A., Tao, Y., Gu, J., Cheng, T., Chen, X., Zhang, X., Zhao, W.: „Thermorph: Democratizing 4D printing of self-folding materials and interfaces", Proceedings of the $2018 \mathrm{CHI}$ conference on human factors in computing systems 2018, (Association for Computing Machinery: New York, 2018), pages 1-12. doi: 10.1145/3173574.3173834.

[3] Guanyun, W., Yang, H., Yan, Z., Ulu, N. G., Tao, Y., Gu, J., Kara, L. B., Yao, L.: "4DMesh: 4D printing morphing non-developable mesh surfaces", Proceedings of the 31st Annual ACM Symposium on User Interface Software and Technology 2018, (Association for Computing Machinery: New York, 2018), pages 623-635. doi: 10.1145/3242587.3242625.

[4] João, F., Deus, A. M., Reis, L., Vaz, M. F., Leite, M.: "Study of the influence of 3D printing parameters on the mechanical properties of PLA", Proceedings of the 3rd International Conference on Progress in Additive Manufacturing 2018, (Pro-AM: Singapore, 2018).

[5] Kadir, G., Türkmen, H. S.: "Common FDM 3D Printing Defects", Proceedings of the 3rd International Congress On 3d Printing Technologies And Digital Industry 2018, (3D-PTC2018: Antalya, 2018), pages 368-370.

[6] Kačergis, L., Rytis, M., Michael, S.: "Influence of fused deposition modeling process parameters on the transformation of 4D printed morphing structures", Smart Materials and Structures 28 (10), 105042, 2019. doi: 10.1039/C7MH00269F. doi:10.1088/1361-665X/ab3d18.

[7] Penjumras, P., Rahman, A.R., Talib, R. A., Abdan, K.: "Mechanical properties and water absorption behaviour of durian rind cellulose reinforced poly (lactic acid) biocomposites", International Journal on Advanced Science Engineering and Information Technology 5, 343-349, 2015. doi: 10.18517/ijaseit.5.5.574.

[8] Ravichandra, R. A., Shanmugam, K.: "Additive manufacturing-enabled shape transformations via FFF 4D printing", Journal of Materials Research 33 (24), 4362-4376, 2018. doi: 10.1557/jmr.2018.397.

[9] Shie, M.Y., Shen, Y.F., Astuti, S.D., Lee, A.K.-X., Lin, S.-H., Dwijaksara, N.L.B., Chen, Y.-W.: "Review of Polymeric Materials in 4D Printing Biomedical Applications", Polymers (Basel) 11 (11), 17, 2019. doi: 10.3390/polym11111864.

[10] Singh, K. M.: "Design and development of a system to detect defects in 3D printing through analysis of layer images", PhD thesis, Politecnico di Torino, 2019.

[11] Song, R., Cassandra, T.: "Material waste of commercial FDM printers under realstic conditions", Proceedings of the 26th Annual International Solid Freeform Fabrication 2016, Proceedings of the 27th Annual International Solid Freeform Fabrication Symposium- An Additive Manufacturing Conference, pp. 1217-1229.

[12] Teunis, V. M., Janbaz, S., Zadpoor, A. A.: "Programming 2D/3D shape-shifting with hobbyist 3D printers", Materials horizons 4 (6), 1064-1069, 2017. doi: 10.1039/c7mh00269f.

[13] Tham, W. L., Poh, B. T., Ishak, Z. A. M., Chow, W. S.: "Characterisation of water absorption of biodegradable poly (lactic acid)/halloysite nanotube nanocomposites at different temperatures", Journal of Engineering Science 12, 13, 2016.

[14] Uzir, W. M., Hassan, A., Ibrahim, A. N., Zawawi, N. A., Kunasegeran, K.: "Mechanical, thermal and chemical resistance of epoxidized natural rubber toughened polylactic acid blends", Sains Malaysiana 44 (11), 1615-1623, 2015.

[15] Valeraga, P. A., Batista, M., Salguero, J., Girot, F.: "Influence of PLA filament conditions on characteristics of FDM parts", Materials 11 (8), 1322, 2018. doi: 10.3390/ma11081322.

[16] Zaldivar, R. J., Mclouth, T. D., Ferrelli, G. L., Patel, D. N., Hopkins, A. R., Witkin, D.: “Effect of initial filament moisture content on the microstructure and mechanical performance of ULTEM ${ }^{\circledR} 9085$ 3D printed parts", Additive Manufacturing 24, 457-466. 2018. doi: 10.1016/j.addma.2018.10.022.

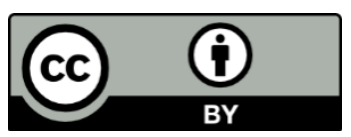

(C) 2020 Authors. Published by the University of Novi Sad, Faculty of Technical Sciences, Department of Graphic Engineering and Design. This article is an open access article distributed under the terms and conditions of the Creative Commons Attribution license 3.0 Serbia (http://creativecommons.org/licenses/by/3.0/rs/). 